\title{
239 Tracheobronchial foreign bodies
}

A Are most common between ages 10 and 15 years.

B May cause an initial episode of choking.

C May present as a unilateral wheeze.

D Can cause a haemoptysis.

E Become symptomatic within a few days.

\section{In the management of an inhaled foreign body}

A The chest $X$-ray is always abnormal if the site of impaction is the bronchus.

B Conservative management should be employed initially.

C A combined bronchoscope and grasping forceps is useful for soft vegetable foreign bodies.

D The Clerf-Arrowsmith forceps are useful for removing peanuts.

E Steroids should always be administered post removal.

\section{Inflammatory processes in the tracheobronchial tree}

A Tracheitis sicca may be improved by laryngectomy.

$B$ Dextrocardia may rarely be present.

C Acute laryngotracheobronchitis is more common in boys.

D Antitoxins are not required if tracheal diphtheria is treated with systemic penicillin.

E Foreign bodies should always be excluded. 